\title{
Concatenated Beam- and Antenna-domain Layered Belief Propagation for Large MIMO Detection
}

\author{
Takumi Takahashi ${ }^{*}$, Antti Tölli ${ }^{\dagger}$, Shinsuke Ibi ${ }^{\ddagger}$, and Seiichi Sampei * \\ * Department of Information and Communications Technology, Osaka University, Yamada-oka 2-1, Suita 565-0871, Japan \\ $\dagger$ Centre for Wireless Communications (CWC), FI-90014 University of Oulu, Finland \\ ¥ Faculty of Science and Engineering, Doshisha University, 1-3 Tataramiyakodani, Kyotanabe-shi, 610-0394, Japan \\ Email: *\{takahashi, sampei\}@comm.eng.osaka-u.ac.jp, †antti.tolli@oulu.fi, †sibi@mail.doshisha.ac.jp
}

\begin{abstract}
This paper proposes concatenated beam- and antenna-domain belief propagation (BP) based signal detector for realizing low-complexity large multi-user multi-input multioutput (MU-MIMO) detection. As a low-complexity strategy for large-scale MU detection (MUD), group-specific signal detection in the beam domain has been proposed, where user equipments (UEs) are grouped based on the long-term channel statistics at a base station (BS) to design outer beamformer (OBF). In this case, the BP-based algorithm is effective as the subsequent signal detector to compensate for the performance degradation due to the dimensionality reduction caused by the OBF. However, the convergence characteristics of beam-domain $B P$ is degraded as compared to the antenna-domain BP due to increased correlation between the beliefs. To avoid the impairments, we propose a novel concatenated structure of beam- and antenna-domain BP layers, which allows us to benefit from the advantages of both domains while maintaining low signal processing complexity. Numerical results show the validity of our proposed method in terms of the bit error rate (BER) performance both in the coded and uncoded cases and the computational complexity.
\end{abstract}

\section{INTRODUCTION}

Large-scale multi-input multi-output (MIMO) systems that employ a large number of transmit and receive antennas can simultaneously provide a high degree of spatial multiplexing gain and improved detection reliability [1]. In particular, uplink multi-user MIMO (MU-MIMO) aimed at supporting a massive amount of simultaneous wireless communications has attracted an increasing amount of attention [2]-[4]. Under such scenarios, large-scale MU detection (MUD) in antennadomain becomes computationally expensive owing to highdimensional matrix operations even if linear spatial filters such as minimum mean square error (MMSE) filter are utilized [2], [3].

To reduce such a computational cost, fully digital twostage signal processing consisting of slowly varying outer beamfomer (OBF) and group-specific MUD for fast channel variations is proven effective [5]. The idea of this approach dates back to joint spatial division and multiplexing (JSDM) [6] for downlink scenarios, where user equipments (UEs) are grouped based on similar transmit correlation matrices to design outer beamformer (OBF) [6]-[9]. Similarly for uplink, the angular spread of receive waves is naturally limited based on the local scatters around the UEs, thus, their energy is concentrated in a subspace of the beam-domain channel. By exploiting the approximate sparsity, UE grouping can be easily conducted based on matching angular spread. The OBF for each group is constructed by selecting beams based on the long-term channel statistics and used to efficiently reduce the dimensions of beam-domain channel [6]-[9]. Consequently, the computational cost of subsequent signal detection on the reduced beam-domain channel is significantly reduced. However, the dimensionality reduction also results in the performance degradation of MUD, due to incompleteness of UE grouping (over-lapping angular spread), especially in highspatial loaded MU-MIMO scenarios.

To improve the performance while reducing the computational cost owing to the OBF, layered BP was proposed in [5], which has a concatenated structure of two different BP layers: MMSE-based BP [10]-[12] layer and matched filter (MF) based BP [2], [13] layer. Each layer has a different role, and this clear role-sharing allows us to efficiently suppress intraand inter-group interference in stages. However, when the MFbased BP typified by approximate message passing (AMP) [13] and Gaussian BP (GaBP) [2] is performed in the beam domain, its convergence property is degraded as compared to antenna-domain MF-based BP. This is due to the fact that the receive beamformer biases and partially enhances the instantaneous correlation between beliefs exchanged in each iteration process. Unfortunately, this degradation becomes more severe when the channel decoder is utilized as the post-stage process of the BP-based signal detection, due to model error of the output log likelihood ratios (LLRs).

As a simple and highly effective solution for this problem, we consider beam-antenna domain conversion in the concatenated structure of layered BP detector. That is, while the strongly correlated intra-group interference is suppressed in the beam domain, the remaining interference is removed by MF-based BP in the antenna domain. This domain conversion allows us to benefit simultaneously from the advantages of antenna- and beam-domain processing. The resultant output LLR is also generated in the antenna domain.

The remainder of this paper is organized as follows. As a preliminary step, Sec. II presents mathematical notations and a system model. The OBF design using the long-term channel statistics and the resultant beam-domain signal model is also presented. Sec. III presents beam-antenna domain conversion in layered belief propagation. Sec. IV validates the proposed method through computer simulations both in the uncodedand coded-case, as well as brief complexity analysis. Finally, Sec. V concludes the paper with a summary.

Throughout this paper, $P_{\mathrm{a} \mid \mathrm{b}}[a \mid b]$ and $p_{\mathrm{a} \mid \mathrm{b}}(a \mid b)$ respectively represent the conditional probability mass function (PMF) and the probability density function (PDF) of a realization $a$ of random variable a given the occurrence of a realization $b$ of 
random variable b. $\mathbb{E}_{\mathrm{a}}\{\cdot\}$ is the expected value of random variable a. $\mathbb{E}_{\mathrm{a} \mid \mathrm{b}=b}\{\cdot\}$ denotes the conditional expectation of random variable a given the occurrence of a realization $b$ of random variable $b$. uniq $\{\mathcal{A}\}$ returns a set which is reconstructed with the unique elements in $\mathcal{A}$ by removing duplicate elements.

\section{Preliminary SteP}

\section{A. Antenna-domain Signal Model}

Consider an uplink MUD system, where the BS has $N$ receive (RX) antennas in uniform linear array (ULA) pattern and $M(\leq N)$ UEs are equipped with a single transmit (TX) antenna. The uniformly distributed UEs around the BS tend to be collocated geographically, and can be naturally clustered into $G$ group based on matching angular spread with $\mathcal{G}=\{1, \ldots, g, \ldots, G\}$ representing the set of UE groups. Let $\mathcal{U}_{g}$ be the set of all UEs assigned to group $g \in \mathcal{G}$, where $\left|\mathcal{U}_{g}\right|=U_{g}$. The set of all UEs is denoted by $\mathcal{U}=\cup_{g \in \mathcal{G}} \mathcal{U}_{g}$, where $|\mathcal{U}|=M=\sum_{g \in \mathcal{G}} U_{g}$.

The $m$-th UE conveys a modulated symbol $x_{m}$, which represents one of $Q$ constellation points $\mathcal{X}=\left\{\chi_{1}, \ldots, \chi_{q}, \ldots, \chi_{Q}\right\}$. The average power density of the constellations in the set $\mathcal{X}$ is denoted by $E_{\mathrm{s}}$. Let $\boldsymbol{x} \in \mathcal{X}^{M \times 1}$ and $\boldsymbol{y} \in \mathbb{C}^{N \times 1}$ denote the TX and RX symbol vectors, respectively. Assuming frequency flat and slow fading environments, $\boldsymbol{y}$ is given by

$$
\boldsymbol{y}=\boldsymbol{H} \boldsymbol{x}+\boldsymbol{z},
$$

where $\boldsymbol{H} \in \mathbb{C}^{N \times M}$ is an $N \times M$ antenna-domain channel matrix, where $\boldsymbol{H}$ is assumed constant during the coherence time $T$. The vector $z \in \mathbb{C}^{N \times 1}$ is a complex additive white Gaussian noise (AWGN) vector, whose entries $z_{n}$ obey $\mathcal{C N}\left(0, N_{0}\right)$, where $N_{0}$ is the noise power spectral density and $\mathbb{E}_{\mathbf{z}}\left\{z z^{\mathrm{H}}\right\}=N_{0} \boldsymbol{I}_{N}$. With the above-mentioned signal model, the conditional PDF of RX vector $\boldsymbol{y}$ is given by

$$
p_{\mathbf{y} \mid \mathbf{x}}(\boldsymbol{y} \mid \boldsymbol{x})=\frac{1}{\left(\pi N_{0}\right)^{N}} \exp \left[-\frac{\|\boldsymbol{y}-\boldsymbol{H} \boldsymbol{x}\|^{2}}{N_{0}}\right] .
$$

As an approximate spatial fading correlation model among RX antennas, the geometric one-ring model [14] is utilized. Assuming diffuse 2-D field of isotropic scatters around the UEs, the $(i, j)$ element of the RX spatial correlation matrix for the $m$-th UE, $\boldsymbol{\Theta}_{m} \in \mathbb{C}^{N \times N}$, is given by

$$
\left[\boldsymbol{\Theta}_{m}\right]_{i, j}=\frac{1}{\Delta \psi_{m}} \int_{\psi_{m}^{\min }}^{\psi_{m}^{\max }} \exp [\mathbf{j} \pi(i-j) \cos (\psi)] d \psi,
$$

which denotes the correlation coefficient between the $i$-th and $j$-th RX antenna elements. Here, waves arrive from the $m$-th UE with an angular spread $\Delta \psi_{m}=\psi_{m}^{\max }-\psi_{m}^{\min }$. The antenna element spacing is fixed to half the wavelength. With the aid of (3), the $m$-th column vector of $\boldsymbol{H}$ is given by

$$
\boldsymbol{h}_{m}=\boldsymbol{\Theta}_{m}^{1 / 2} \boldsymbol{v}_{m},
$$

where each element of $v_{m} \in \mathbb{C}^{N \times 1}$ obeys $C \mathcal{N}(0,1)$.

\section{B. Outer Beamformer Design}

Let $B_{g}$ denotes the number of statistical beams oriented towards each UE group $g \in \mathcal{G}$, where $\sum_{g \in \mathcal{G}} B_{g} \leq N$. The resultant OBF for group $g, \boldsymbol{B}_{g}$, contains $B_{g}$ statistical beams corresponding to the UEs in group $g$. Assuming the UEs are grouped based on their long-term channel statistics, e.g. channel covariance matrix, we can continue to use the groupspecific OBF over multiple channel realization. In [9], discrete Fourier transform (DFT) beam selection was numerically shown to be better than the other selection schemes, therefore, we employ the DFT scheme to find the OBF matrix in this paper. The DFT beam selection use the column vectors of DFT matrix $\boldsymbol{D}=\left[\boldsymbol{d}_{1}, \ldots, \boldsymbol{d}_{N}\right] \in \mathbb{C}^{N \times N}$ to form the OBF matrix [8], [9]. Then, the problem reduces to finding a subset of column vectors from the unitary DFT matrix $\boldsymbol{D}$.

Here, we define $\boldsymbol{H}_{\mathcal{U}_{g}}=\left[\boldsymbol{h}_{\mathcal{U}_{g}(1)}, \ldots, \boldsymbol{h}_{\mathcal{U}_{g}\left(U_{g}\right)}\right]$ as the stacked channel matrix corresponding all UEs in group $g$. From (4), the covariance (correlation) matrix is given by

$$
\boldsymbol{R}_{g}=\mathbb{E}_{\mathrm{V}}\left\{\boldsymbol{H}_{\mathcal{U}_{g}} \boldsymbol{H}_{\mathcal{U}_{g}}^{\mathrm{H}}\right\}=\sum_{i \in \mathcal{U}_{g}} \boldsymbol{\Theta}_{i}
$$

To maximize the energy collected by the statistical beams, we select $B_{g}$ DFT column vectors for each group $g \in \mathcal{G}$ as

$$
\begin{gathered}
\text { Initialization : } \mathcal{D}=\{1, \ldots, N\}, \mathcal{B}_{g}=\emptyset, \\
j=\underset{\underline{n} \in \mathcal{D}}{\arg \max }\left(\boldsymbol{d}_{\underline{n}}^{\mathrm{H}} \boldsymbol{R}_{g} \boldsymbol{d}_{\underline{n}}\right), \forall \underline{n} \in \mathcal{D}, \\
\mathcal{B}_{g}=\mathcal{B}_{g} \cup\{j\}, \mathcal{D}=\mathcal{D} \backslash \mathcal{B}_{g} .
\end{gathered}
$$

Upon finding the subset $\mathcal{B}_{g}$, the resultant OBF matrix is given by $\boldsymbol{B}_{g}=\left[\boldsymbol{d}_{\mathcal{B}(1)}, \ldots, \boldsymbol{d}_{\mathcal{B}\left(B_{g}\right)}\right] \in \mathbb{C}^{N \times B_{g}}$, where $\boldsymbol{B}_{g}^{\mathrm{H}} \boldsymbol{B}_{g}=\boldsymbol{I}_{B_{g}}$.

\section{Beam-domain Signal Model and UE Selection}

For each UE group $g \in G$, the beam-domain RX vector $\boldsymbol{r}_{g} \in \mathbb{C}^{B_{g} \times 1}$ can be represented as

$$
\boldsymbol{r}_{g}=B_{g}^{\mathrm{H}} \boldsymbol{y}=\boldsymbol{\Xi}_{g} \boldsymbol{x}+\boldsymbol{v}_{g},
$$

where $\boldsymbol{\Xi}_{g}=\boldsymbol{B}_{g}^{\mathrm{H}} \boldsymbol{H} \in \mathbb{C}^{B_{g} \times M}$ and $\boldsymbol{v}_{g}=\boldsymbol{B}_{g} \boldsymbol{z} \in \mathbb{C}^{B_{g} \times 1}$ are the beam-domain channel and noise, respectively. Considering the computational cost for computing spatial filters, the number of row dimensions $M$ still remains undesirable: reformulate.

For further dimensionality reduction of the beam-domain channel matrix, we can ignore negligibly small inter-group interference (IGI) based on the attenuated sidelobes of OBF. Similarly to (6), in this case, we select $S_{g}(\leq M)$ UEs for each group $g \in \mathcal{G}$ as

$$
\begin{gathered}
\text { Initialization }: \mathcal{E}=\{1, \ldots, M\}, \mathcal{S}_{g}=\emptyset, \\
j=\underset{\underline{m} \in \mathcal{M}}{\arg \max }\left(\sum_{i \in \mathcal{B}_{g}} \boldsymbol{d}_{i}^{\mathrm{H}} \boldsymbol{\Theta}_{\underline{m}} \boldsymbol{d}_{i}\right), \forall \underline{m} \in \mathcal{D}, \\
\mathcal{S}_{g}=\mathcal{S}_{g} \cup\{j\}, \mathcal{E}=\mathcal{E} \backslash \mathcal{S}_{g} .
\end{gathered}
$$

By ignoring IGI from the UEs not included in the subset $\mathcal{S}_{g}$, the beam-domain channel size can be reduced to $B_{g} \times S_{g}$.

\section{BEAM-ANTENNA DOMAIN CONVERSION IN LAYERED BELIEF PROPAGATION}

In this section, we present the proposed antenna-beam domain conversion scheme after reviewing the typical layered BP detector to clarify the ill-convergence issue in beamdomain MF-based BP. To simplify the mathematical notations, we assume quadrature phase shift keying (QPSK) signaling $\left(\mathcal{X}=\left\{ \pm w_{x} \pm \mathrm{j} w_{x}\right\}, w_{x}=\sqrt{E_{\mathrm{s}} / 2}, \mathrm{j}=\sqrt{-1}\right)^{1}$.

\footnotetext{
${ }^{1}$ The layered BP proposed in this paper can be extended to QAM signaling after some appropriate mathematical manipulations [15]
} 


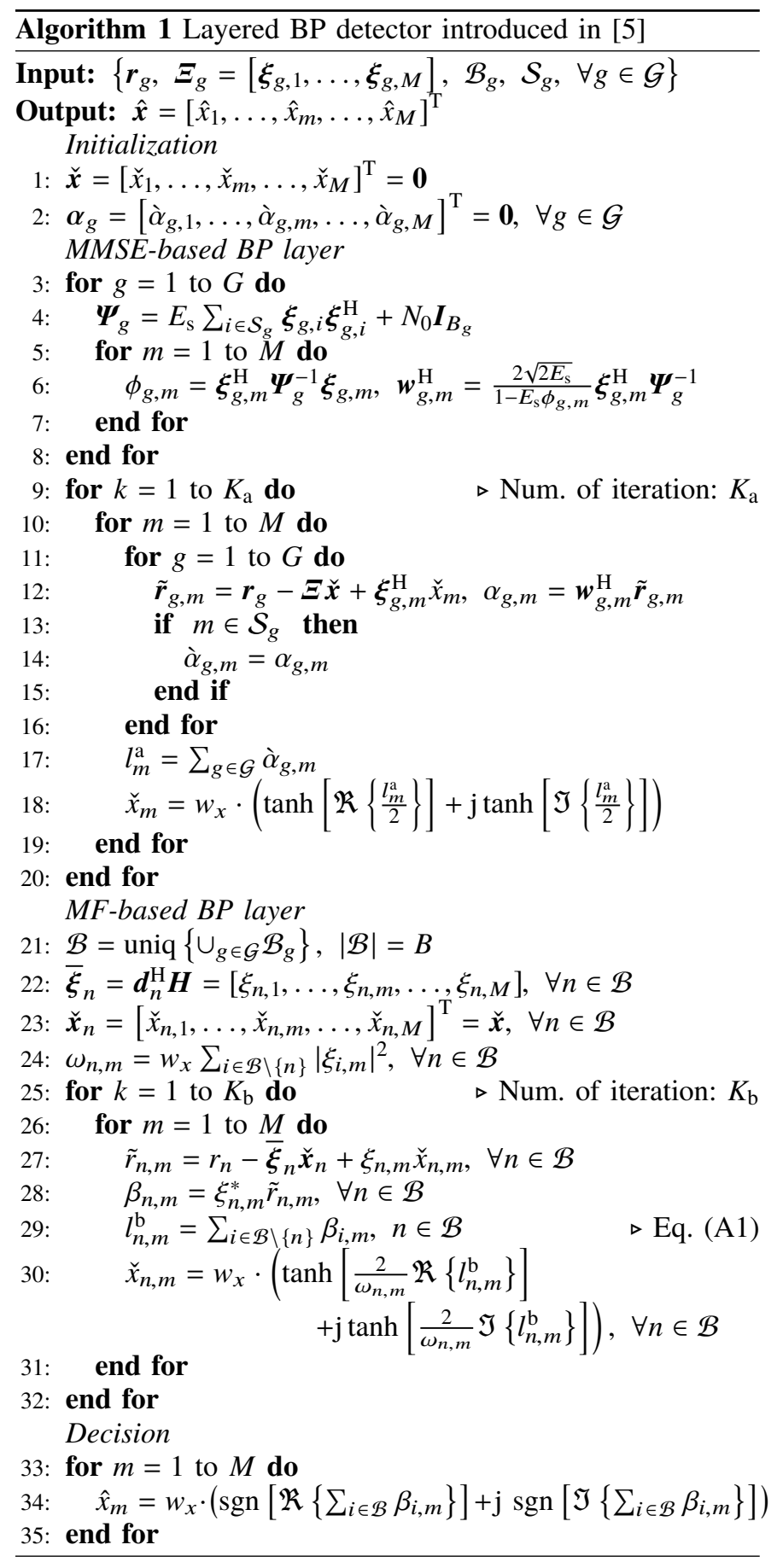

\section{A. Problem Statements}

The pseudo-code of layered BP introduced in [5] is provided in Algorithm 1. For convenience, the node selection method [16] is omitted. It consists of two different layers: MMSEbased BP layer and MF-based BP layer, and the concatenated structure can efficiently suppress intra- and inter-group interference in stages. The group-specific MMSE-based BP layer suppresses strongly correlated intra-group interference and strong IGI from UEs selected in (8). On the other hand, the subsequent beam-specific MF-based BP layer prompts convergence of iterative detection by suppressing the remaining

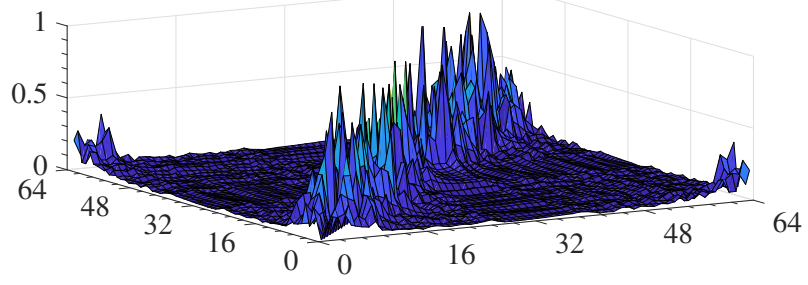

(a) Intensity of the off-diagonal elements in $\boldsymbol{G}_{\Xi}$ normalized by $\boldsymbol{M}$.

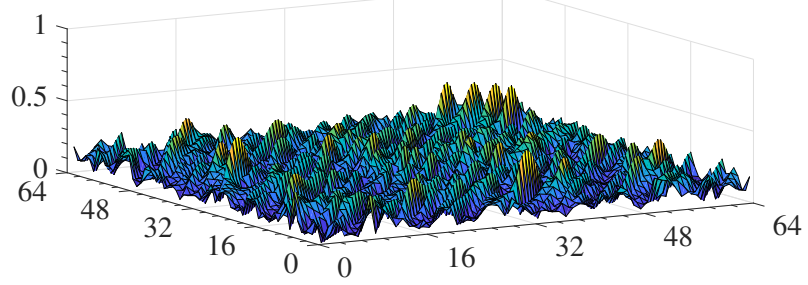

(b) Intensity of the off-diagonal elements in $\boldsymbol{G}_{\mathbf{H}}$ normalized by $\boldsymbol{M}$.

Fig. 1. Intensity of the off-diagonal elements normalized by $M$, where $\left(G, B_{g}, S_{g}\right)=(8,8,16)$ in $(N, M)=(64,48)$ MU-MIMO configuration.

interference induced by group-specific processing. Note that all processes are performed in the beam domain.

To suppress the remaining interference by a maximum ratio combining (MRC) of (A1) in Algorithm 1, the effective noise included in $\beta_{1, m}, \ldots, \beta_{\mathcal{B}(B), m}$ should be uncorrelated as much as possible. When averaging the impact of TX symbols, it deeply depends on the instantaneous correlation of the beam-domain channel coefficients among statistical beams $(1, \ldots, \mathcal{B}(B))$, and can be visualized using the off-diagonal elements in a Gram matrix:

$$
\boldsymbol{G}_{\boldsymbol{\Xi}}=\boldsymbol{\Xi} \boldsymbol{\Xi}^{\mathrm{H}}=\boldsymbol{D}^{\mathrm{H}} \boldsymbol{H} \boldsymbol{H}^{\mathrm{H}} \boldsymbol{D}=\boldsymbol{D}^{\mathrm{H}} \boldsymbol{G}_{\mathbf{H}} \boldsymbol{D},
$$

where $\boldsymbol{\Xi}=\left[\overline{\boldsymbol{\xi}}_{1}^{\mathrm{T}}, \ldots, \overline{\boldsymbol{\xi}}_{N}^{\mathrm{T}}\right]^{\mathrm{T}}=\boldsymbol{D}^{\mathrm{H}} \boldsymbol{H}$ is the beam-domain channel matrix, and $\boldsymbol{G}_{\mathbf{H}}$ is the Gram matrix of antenna-domain channel matrix $\boldsymbol{H}$. That is, the approximation error induced in (A1) becomes more severe as the intensity of the offdiagonal elements in the Gram matrix increases, resulting in ill-convergence behavior of iterative detection.

Fig. 1 shows the intensity of the off-diagonal elements in $\boldsymbol{G}_{\mathbf{H}}$ and $\boldsymbol{G}_{\boldsymbol{\Xi}}$ normalized by $\boldsymbol{M}$, respectively. As can be seen in Fig. 1(a), the RX beamformer $\boldsymbol{D}^{\mathrm{H}}$ behaves just like a correlation matrix, and intensity of the off-diagonal elements in $\boldsymbol{G}_{\boldsymbol{\Xi}}$ is regularly biased and partially enhanced. In particular, the adjacent beams in the azimuthal direction increase the instantaneous correlation of the beam-domain channel coefficients, resulting in the strong off-diagonal elements around the diagonal elements. Consequently, noise enhancements due to the instantaneous correlation are induced in MRC of (A1), and error propagation tends to occur. On the other hand, in Fig. 1(b), intensity of the off-diagonal elements in $\boldsymbol{G}_{\mathbf{H}}$ is uniformly distributed, and suppressed to small values as compared to that in $\boldsymbol{G}_{\boldsymbol{\Xi}}$ in the beam domain. Thus, by performing MF-based BP including MRC in the antenna domain, we can improve the convergence property. Note that this ill-convergence issue in beam-domain MF-based BP does not occur in MMSE-base $\mathrm{BP}$ [12] owing to the belief combining via an inverse matrix. 


\section{B. Antenna-domain MF-based BP layer}

From the beam-domain channel $\boldsymbol{\Xi}$, the antenna-domain channel is constructed via DFT transform as $\boldsymbol{H}=\boldsymbol{D} \boldsymbol{\Xi}$. The incremental computational cost for this operation is only $O\left(M N \log _{2} N\right)$ per channel realization. The process flow is basically the same as in [16] except for the LLR computations at the final iteration.

First, the soft interference cancellation (soft IC) for the $n$ th antenna-domain RX symbol $\boldsymbol{y}_{n}$, is carried out using a soft symbol vector $\check{\boldsymbol{x}}_{n}=\left[\check{x}_{n, 1}, \ldots, \check{x}_{n, m}, \ldots, \check{x}_{n, M}\right]^{\mathrm{T}}$. At the first iteration process $(k=1), \check{\boldsymbol{x}}_{n}=\breve{\boldsymbol{x}}, \forall n \in \mathcal{N}=\{1, \ldots, N\}$. For detecting $x_{m}, \exists m \in \mathcal{M}=\{1, \ldots, M\}$, the cancellation process is expressed as

$$
\begin{aligned}
\tilde{y}_{n, m} & =y_{n}-\overline{\boldsymbol{h}}_{n} \check{\boldsymbol{x}}_{n}+h_{n, m} \check{x}_{n, m} \\
& =h_{n, m} x_{m}+\sum_{i \in \mathcal{M} \backslash\{m\}} h_{n, i}\left(x_{i}-\check{x}_{i}\right)+z_{n}, \quad \forall n \in \mathcal{N},
\end{aligned}
$$

where $\overline{\boldsymbol{h}}_{n}=\left[h_{n, 1}, \ldots, h_{n, m}, \ldots, h_{n, M}\right]$ denotes the $n$-th row vector of $\boldsymbol{H}$. For reducing the computational cost, MF output is utilized as a belief, instead of traditional LLR, as

$$
\gamma_{n, m}=h_{n, m}^{*} \tilde{y}_{n, m}, \forall n \in \mathcal{N} \text {. }
$$

Assuming that equivalent noise included in $\gamma_{1, m}, \ldots, \gamma_{N, m}$ are not correlated, in BP regime [13], a prior belief is provided by an extrinsic value to avoid self-noise regression, as

$$
l_{n, m}^{\mathrm{c}}=\sum_{i \in \mathcal{N} \backslash\{n\}} \gamma_{i, m}, \forall n \in \mathcal{N} .
$$

With the aid of channel hardening effect [2], the conditional expectation of TX symbol $x_{m}$ is approximately computed by the following activation function: [16]

$$
\begin{aligned}
\check{x}_{n, m} & =f\left(l_{n, m}^{\mathrm{c}}\right) \\
& =w_{x} \cdot\left(\tanh \left[\frac{\sigma}{\mu_{n, m}} \mathfrak{R}\left\{l_{n, m}^{\mathrm{c}}\right\}\right]+\mathrm{j} \tanh \left[\frac{\sigma}{\mu_{n, m}} \mathfrak{J}\left\{l_{n, m}^{\mathrm{c}}\right\}\right]\right),
\end{aligned}
$$

where we define the effective gain of $l_{n, m}^{\mathrm{c}}$ :

$$
\mu_{n, m}=w_{x} \sum_{i \in \mathcal{N} \backslash\{n\}}\left|h_{i, m}\right|^{2} .
$$

$\sigma$ is the predetermined design parameter; in the simulations, we set $\sigma=2.0$. When the number of iterations in MF-based BP reaches the predetermined value $K^{\mathrm{b}}, x_{m}$ is detected by hard decision of $\sum_{i \in \mathcal{N}} \gamma_{i, m}$.

When using the channel decoder as the post-stage processing, the LLR belief should be computed as the output of MFbased BP layer at the final iteration. By approximating the second and third terms in (10) as a complex Gaussian random variable $\phi_{n, m} \sim \mathcal{C N}\left(0, \psi_{n, m}\right)$, a complex-valued LLR for Gray coded QPSK symbol $x_{m}$ at each RX antenna $n$ is computed according to the definition [1] as

$$
\zeta_{n, m}=2 \sqrt{2 E_{\mathrm{s}}} \cdot h_{n, m}^{*} \tilde{y}_{n, m} / \psi_{n, m} .
$$

where we have

$$
\begin{aligned}
\psi_{n, m} & =\mathbb{E}_{\mathrm{Z}_{n},\left\{x_{i}, \forall i \in \mathcal{M} \backslash\{m\}\right\} \|_{n}^{\mathrm{c}}=\left[l_{n, 1}^{\mathrm{c}}, \ldots, l_{n, M}^{\mathrm{c}}\right]}\left\{\phi_{n, m} \phi_{n, m}^{*}\right\} \\
& =\overline{\boldsymbol{h}}_{n} \boldsymbol{\Delta}_{n, m} \overline{\boldsymbol{h}}_{n}^{\mathrm{H}}+N_{0}, \\
\boldsymbol{\Delta}_{n, m} & =\operatorname{diag}\left[\delta_{n, 1}, \ldots, \delta_{n, m-1}, 0, \delta_{n, m+1}, \ldots, \delta_{n, M}\right], \\
\delta_{n, m} & =E_{\mathrm{S}}-\left|\check{x}_{n, m}\right|^{2} .
\end{aligned}
$$

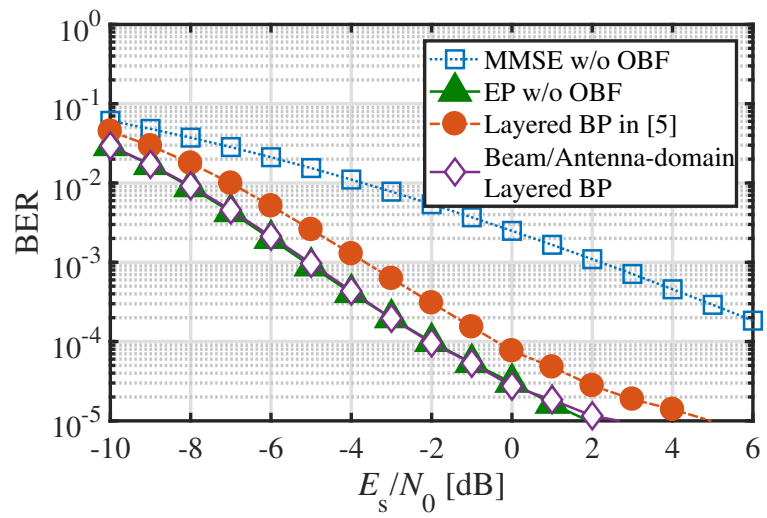

Fig. 2. BER performances in uncoded case: $(N, M)=(64,48), U_{g}=$ $M / G=6, \forall g \in \mathcal{G},\left(K_{\mathrm{a}}, K_{\mathrm{b}}\right)=(8,8)$. The number of EP iteration is 8 .

Finally, the output LLR for $x_{m}$ is given by

$$
\lambda_{n, m}=\sum_{i \in \mathcal{N}} \zeta_{i, m} .
$$

In simulations, the NS method [16] is utilized for suppressing the harmful impacts of spatial fading correlation. In this paper, the number of sets is 4 as in the case of [5].

\section{Numerical Results}

\section{A. BER performances}

Computer simulations were conducted to validate the beamantenna domain conversion in layered BP for large-scale MUD. The average RX power from each TX antenna is assumed to be identical on the basis of slow TX power control. 64 sector antennas with 120 degrees aperture are considered. The UEs are naturally partitioned into $G$ segments with $M / G$ UEs randomly dropped in each segment. The angular spread for each UE is 15 degrees. The modulation scheme is Graycoded QPSK. Time and frequency synchronization between TX and RX are assumed to be perfect.

Fig. 2 shows the bit error rate (BER) performances with the system parameters $\left(G, B_{g}, S_{g}\right)=(8,8,16), \forall g \in \mathcal{G}$ in uncoded $(N, M)=(64,48)$ MU-MIMO configurations. As a baseline performance, the curve of typical linear MMSE filter without the use of OBF "MMSE w/o OBF" is drawn. As a comparison with the state-of-the-art expectation propagation (EP) detector, "EP w/o OBF" performance is also presented ${ }^{2}$. Note that the computational complexity is prohibitive in large-scale MUD.

Compared to "MMSE w/o OBF", layered BP in [5] can significantly improve the detection capability despite of extremely low-complexity owing to the concatenated structure. By extending this approach to beam-antenna domain conversion, further improvements can be achieved. The proposed "Beam/Antenna-domain Layered BP" enables to further obtain about $1.8 \mathrm{~dB}$ improvement, and can approach "EP w/o OBF".

Let us shift our focus to the BER performances of Fig. 3 in coded MU-MIMO systems. The system parameters are the same as the uncoded case. The low-density parity-check (LDPC) code of rate 2/3 and length 1944 bits used in the

\footnotetext{
${ }^{2}$ The EP detector used in this paper is different from that used in [5]. Some approximations based on the large system limit in [12] are removed to improve the convergence property in exchange for the additional computational cost.
} 


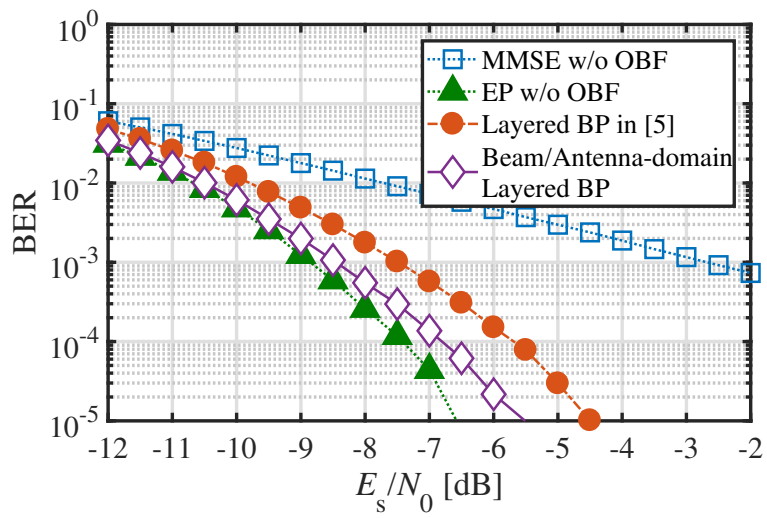

Fig. 3. BER performances in coded case: $(N, M)=(64,48), U_{g}=M / G=$ $6, \forall g \in \mathcal{G},\left(K_{\mathrm{a}}, K_{\mathrm{b}}\right)=(8,8)$. The number of EP iteration is 8 .

TABLE I

COMPUTATIONAL COMPARISON OF DIFFERENT DETECTORS

\begin{tabular}{|c|c|c|}
\hline Detector & For each channel realization & For each TX vector \\
\hline MMSE w/o OBF & $O\left(M^{2} N+M^{3}\right)$ & $O(M N)$ \\
\hline EP w/o OBF & $O\left(M^{2} N+M^{3}\right)$ & $O\left(K\left(M^{2} N+M^{3}\right)\right)$ \\
\hline MMSE w/ OBF & $O\left(G B_{g} S_{g}\right)$ & $O(M N)$ \\
\hline Layered BP in [5] & $O\left(G B_{g} S_{g}\right)$ & $O\left(\left(K^{\mathrm{a}}+K^{\mathrm{b}}\right) M N\right)$ \\
\hline $\begin{array}{c}\text { Beam/Antenna-domain } \\
\text { Layered BP }\end{array}$ & $O\left(G B_{g} S_{g}+M N \log _{2} N\right)$ & $O\left(\left(K^{\mathrm{a}}+K^{\mathrm{b}}\right) M N\right)$ \\
\hline
\end{tabular}

IEEE 802.11n standard is applied as the channel code. The error correction by the channel decoder is conducted only once after layered BP iterative detection.

"Layered BP" can obtain large gain as compared to "MMSE w/o OBF", however, there is still non-negligible gaps from "EP w/o OBF" performance. This is due to the fact that the correlation between beliefs caused by the DFT beamformer degrades the convergence property of beam-domain MF-based BP layer, and the model error of output LLRs is induced. The most attractive feature is that the proposed beam-antenna domain conversion allows the layered BP detector to avoid the convergence issue with the beam-domain MF layer and to approach "EP w/o OBF" performance without significant additional computational cost as compared to [5]. More specifically, the degradation is only about $0.5 \mathrm{~dB}$ at $\mathrm{BER}=10^{-4}$ in the present large MU-MIMO configuration.

\section{B. Complexity analysis}

Table I summarizes the order of complexities. Layered BP detector can reduce the computational cost to a few percents of conventional MMSE-based BP detectors typified by EP [12] with a minimal reduction in the detection capability [5]. When we employ the proposed beam-antenna domain conversion, the computational cost of layered BP per channel realization can be approximated to $O\left(M N \log _{2} N\right)$ because $M N \log _{2} N \gg$ $G B_{g} S_{g}$. Even in this case, the complexity remains roughly $7 \%$ of that of the EP detector in $(N, M)=(64,48)$ configuration. Moreover, the computational cost for detecting each TX vector is not changed and is less than $2 \%$ complexity of EP.

\section{CONClusion}

In this paper, we proposed beam-antenna domain conversion in the layered BP detector for large-scale MUD based on the
OBF. Although the beam-domain BP-based MUD allows us to significantly reduce the computational cost while maintaining near-optimal performance, the convergence property of iterative detection is degraded when the MF-based BP is utilized in the beam domain. The off-diagonal elements in the Gram matrix of beam-domain channel are significantly enhanced by the DFT beamformer, leading to severe instantaneous correlation among beliefs. To avoid such impairment, domain conversion is introduced in the concatenated structure of layered BP detector. By performing the MF-based layer in the antenna domain, the advantages of antenna- and beam-domain processing are achieved simultaneously. The computer simulations confirm that the proposed beam- and antenna-domain low-complexity detector can approach near EP performance even in coded MU-MIMO scenarios with high spatial load.

\section{ACKNOWLEDGEMENT}

This work was financially supported by JSPS KAKENHI Grant Number JP18H03765, Japan.

\section{REFERENCES}

[1] L. Hanzo, O. Alamri, M. El-Hajjar, and N. Wu, Near-Capacity MultiFunctional MIMO Systems Sphere-Packing, Iterative Detection and Cooperation. Wiley-IEEE Press, May 2009.

[2] A. Chockalingam and B. S. Rajan, Large MIMO Systems. Cambridge University Press, 2014.

[3] S. Yang and L. Hanzo, "Fifty years of MIMO detection: The road to large-scale MIMOs," IEEE Commun. Surveys Tutorials, vol. 17, no. 4, pp. 1941-1988, Fourthquarter 2015.

[4] C. Bockelmann, N. Pratas, H. Nikopour, K. Au, T. Svensson, C. Stefanovic, P. Popovski, and A. Dekorsy, "Massive machine-type communications in 5G: physical and MAC-layer solutions," IEEE Commun. Mag., vol. 54, no. 9, pp. 59-65, Sept. 2016.

[5] T. Takahashi, A. Tölli, S. Ibi, and S. Sampei, "Layered belief propagation for low-complexity large MIMO detection based on statistical beams," in Proc. IEEE ICC 2019, Shanghai, China, May. 2019.

[6] A. Adhikary, J. Nam, J. Ahn, and G. Caire, "Joint spatial division and multiplexing The large-scale array regime," IEEE Trans. Inf. Theory, vol. 59, no. 10 , pp. 6441-6463, Oct 2013

[7] J. Nam, A. Adhikary, J. Ahn, and G. Caire, "Joint spatial division and multiplexing: Opportunistic beamforming, user grouping and simplified downlink scheduling," IEEE Journal of Selected Topics in Signal Processing, vol. 8, no. 5, pp. 876-890, Oct. 2014.

[8] A. Arvola, A. Tölli, and D. Gesbert, "Two-layer precoding for dimensionality reduction in massive MIMO," in 2016 24th European Signal Processing Conf. (EUSIPCO), Aug 2016, pp. 2000-2004.

[9] A. Padmanabhan and A. Tölli, "An iterative approach for inter-group interference management in two-stage precoder design," in Proc. IEEE Globecom 2018, Abu Dhabi, UAE, Dec. 2018.

[10] S. Yang, T. Lv, R. G. Maunder, and L. Hanzo, "From nominal to true a posteriori probabilities: An exact bayesian theorem based probabilistic data association approach for iterative MIMO detection and decoding," IEEE Trans. Commun., vol. 61, no. 7, pp. 2782-2793, July 2013.

[11] J. Ćespedes, P. M. Olmos, M. Sánchez-Fernández, and F. Perez-Cruz, "Expectation propagation detection for high-order high-dimensional MIMO systems," IEEE Trans. Commun., vol. 62, no. 8, pp. 2840-2849, Aug. 2014.

[12] K. Takeuchi, "Rigorous dynamics of expectation-propagation-based signal recovery from unitarily invariant measurements," Jan. 2017. [Online]. Available: http://arxiv.org/abs/1701.05284

[13] D. L. Donoho, A. Maleki, and A. Montanari, "Message-passing algorithms for compressed sensing," Proc. Nat. Acad. Sci., vol. 106, no. 45, pp. 18914-18 919, Nov. 2009.

[14] W. C. Jakes, Microwave Mobile Communications, 1st ed. Wiley-IEEE Press, 1994.

[15] T. Takahashi, S. Ibi, and S. Sampei, "Design of adaptively scaled belief in multi-dimensional signal detection for higher-order modulation," IEEE Trans, Commun., vol. 67, no. 3, pp. 1986-2001, Mar. 2019.

[16] _ "Design of criterion for adaptively scaled belief in iterative large MIMO detection," IEICE Trans. Commun., vol. E102.B, no. 2, pp. 285297, 2019. 